\title{
Using Focus + Context Techniques to Visualize Building Information Model in virtual Geo-Environment
}

\author{
YING Shen ${ }^{\text {a,b }}$, LI Chengpeng ${ }^{\mathrm{a}, *}$, LI Weiyang ${ }^{\mathrm{a}}$, CHEN Naibin ${ }^{\mathrm{a}}$, ZHAO Zhigang ${ }^{\mathrm{c}}$ \\ ${ }^{a}$ School of Resource and Environmental Sciences, Wuhan University, Wuhan 430079, China . LI Chengpeng \\ lichengpeng@whu.edu.cn, YING Shen shy@whu.edu.cn, LI Weiyang hzauqincheng@aliyun.com, CHEN Naibin \\ Chen_nb@whu. edu.cn \\ ${ }^{b}$ Key Lab of Geographic Information System, Educational Ministry, Wuhan University, Wuhan 430079, China \\ ${ }^{c}$ Research Institute for Smart City, Shenzhen University, Shenzhen 518060, China; ZHAO Zhigang zhaozgRISC@ szu.edu.cn \\ * Corresponding author: LI Chengpeng lichengpeng@whu. edu. cn
}

Keywords: Building Information Model, Geo-Environment, Focus + context visualization

The Integration of BIM and GIS is conducive to urban planning, construction and management, and have explored by relevant researchs (Yeo et al., 2016; Gilbert et al.; Wang et al.). From the beginning, BIM researchers thought that GIS could represent the building environment partially, while GIS researchers considered that BIM could only provide a detail data model about the buildings as one of GIS data sources. With deepening the technique integration and broadening the applications, it become a trend to integrate BIM and GIS, and both semantics and geometry between them are feasible to interlink and interact (Zhu et al., 2019; Deng et al., 2016). In this case, the paper studies the visualization method and the visual effects of BIM model (with IFC format as input) in virtual geographic environment (VGE), in order to improve human interaction and cognitive ability of BIM model in VGE. Based on Focus + Context techniques (Correa et al., 2016; Bjork et al., 2000; Carpendale et al., 1996), current development focuses on the solutions to the following problems.

\section{1) Focused BIM model in geographic environment.}

During the visualization process, BIM model has serious occlusion problems in two level: group level and component (sub-group) level. The group level occlusion means BIM model as an integral object may be occluded by other buildings in VGE (Qu et al., 2016; Deng et al., 2016; Johansson et al., 2015), and at the component level, inner components inside the BIM model may be occluded by other outer components when we focus or select inner one (Motamedi et al., 2014). So, there are corresponding component-level and group-level focus + context visualization.

In VGE, buildings are important parts, but most of the time, the display of buildings are in the form of block or volume shapes. According to the definition of CityGML, buildings are usually represented by LOD1 or LOD2 objects. However, with the development of BIM technology, more and more requirements are needed to model the focus buildings. The corresponding building information and details are even more abundant than the LOD4 model in CityGML. When BIM model is imported into VGE as a significant rendering object, other buildings that are shown as blocks or volumes are often used as context, so BIM models will be the focus of VGE (Fig 1).

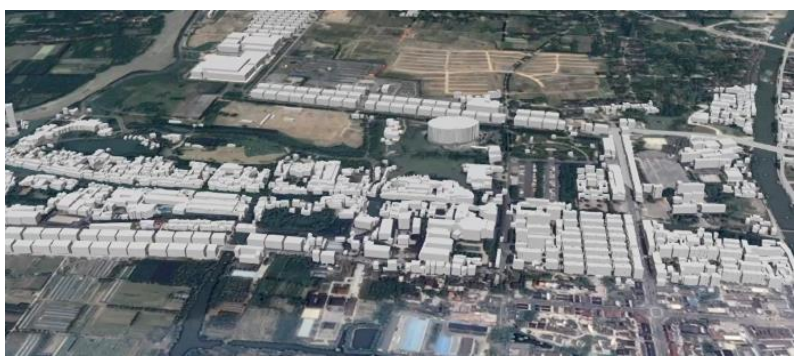

a

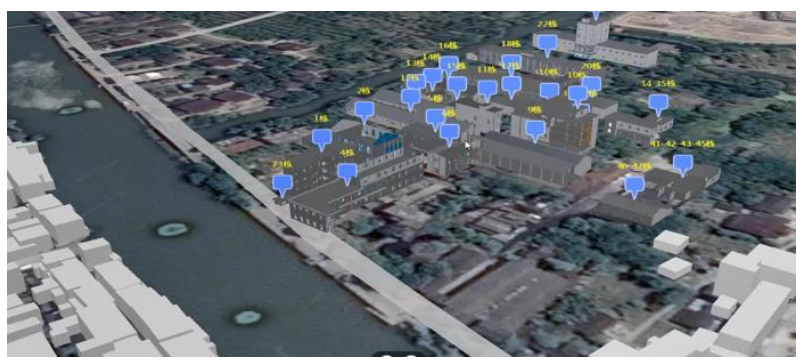

b

Fig.1 BIM Models in VGE (Sample Wuzhen in China): a) VGE; b)building models in VGE

\section{2) Visualization of the internal details of BIM model.}

At the component-level focus + context visualization, conventional visualization software of BIM model use cutaway view, transparent view and explode view to visualize and obtain internal details of the BIM model, but they all have their own limitations.

When we explore BIM model by the cutaway view (Fig2.a), an invisible vertical "knife" is put in the scene and divides the BIM model into two sections. The building part on one side of the section is completely preserved, while the building part on the other side is hidden, which loses the description of the overall relationship and structure of the building. When we visualize the BIM model by transparent view (Fig2.b), the components are highlighted and be semi-transparent, but 
the superposition of the transparent components and the selected opaque components causes the visible confusion. While explode view (Fig2.c) to visualize BIM model is a novel method, but the current software just adjusts each components' position without considering global viewing effects.

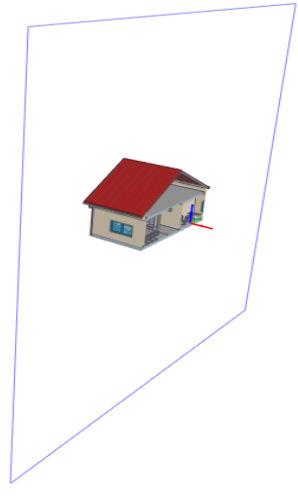

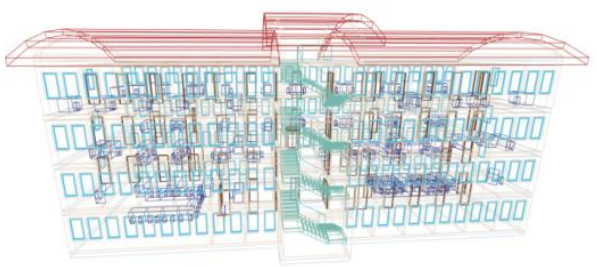

$\mathrm{b}$

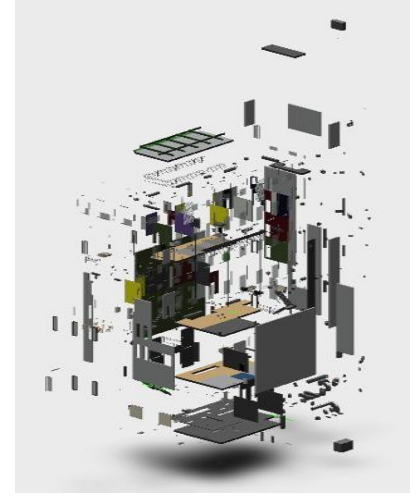

c

Fig. 2 Traditional viewing methods: a) the cutaway view, b) the transparent view, c) the explode view

In the Focus + Context techniques, the corresponding focus visualization method achieves a deformation effect by enlarging, shrinking and shifting the position of focus object (Islam et al., 2007), thus to highlight the focus object and weaken the context. This paper proposes a two-layer Focus + Context visualization method to obtain the inner details of the focus components in BIM model (shown as Fig. 3). Moreover, the key of our researches is that considering the integration of the BIM model visualization in VGE, rather than in BIM model viewing software. In addition, the factors of geographic environment are discussed to improve the visualization effects. The two-layer and two-level (componentlevel and group-level) focus + context visualization (Section 1 and Section 2) should be combined together in VGE to achieve a progressive visualization from macro geographic environment to micro building element environment.

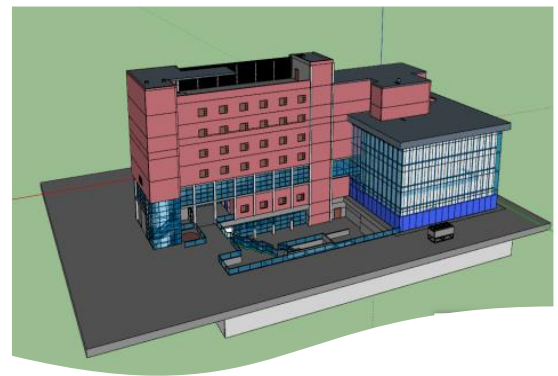

a

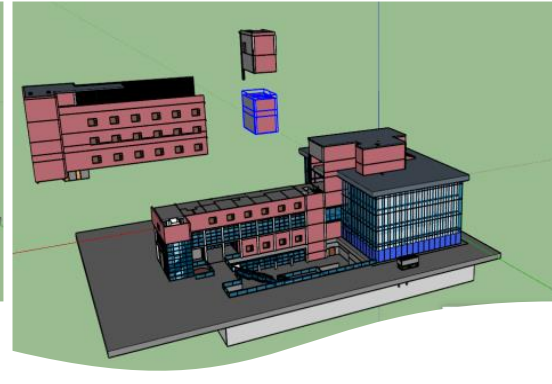

b

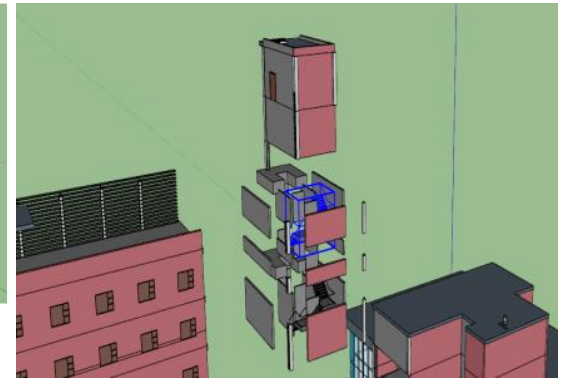

C

Fig.3 Two-layer Focus + Context visualization of the model to see inner details: a) building; b) first-layer focus on building parts; c) second-layer focus on build elements inside the first-layer focus.

\section{3) Visualization of spatial relationship in BIM model.}

Spatial relationships are core elements in GIS. Current BIM models have seldom records and studies on spatial relationships but are necessary (Juszczyk et al., 2016). In geographic environment, it is vital to give the full play to the advantages of BIM models, especially its detail components, and to bound full cycle-life information. So building component-level spatial relationships among BIM models are constructed to integrate spatial analysis. The connectivity relationships among walls, doors and windows in Figure 4 are built as lines between walls to support the indoor navigation and wayfinding.

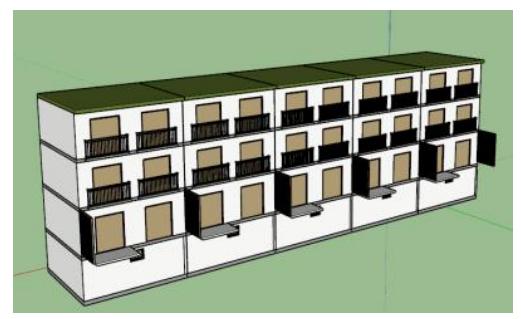

a

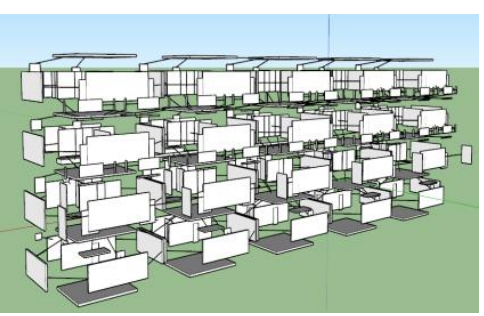

b

Fig.4 The connectivity among walls, doors and windows of the BIM components: a) building; b) lines indicating spatial relationships between walls 


\section{References}

Yeo I A, Yee J J. Development of an automated modeler of environment and energy geographic information (E-GIS) for ecofriendly city planning[J]. Automation in Construction, 2016, 71: 398-413.

Gilbert T, Barr S, James P, et al. Software Systems Approach to Multi-Scale GIS-BIM Utility Infrastructure Network Integration and Resource Flow Simulation[J]. ISPRS International Journal of Geo-Information, 2018, 7(8): 310.

Wang H, Pan Y, Luo X. Integration of BIM and GIS in sustainable built environment: A review and bibliometric analysis[J]. Automation in Construction, 2019, 103: 41-52.

Zhu J, Wang X, Wang P, et al. Integration of BIM and GIS: Geometry from IFC to shapefile using open-source technology[J]. Automation in Construction, 2019, 102: 105-119.

Deng Y, Cheng J C P, Anumba C. Mapping between BIM and 3D GIS in different levels of detail using schema mediation and instance comparison[J]. Automation in Construction, 2016, 67: 1-21.

Correa C, Silver D, Chen M. Illustrative deformation for data exploration[J]. IEEE Transactions on Visualization and Computer Graphics, 2007, 13(6): 1320-1327.

Bjork S, Redstrom J. Redefining the focus and context of focus+ context visualization[C]. IEEE Symposium on Information Visualization 2000. INFOVIS 2000. Proceedings. IEEE, 2000: 85-89.

Carpendale M S T, Cowperthwaite D J, Fracchia F D. Distortion viewing techniques for 3-dimensional data[C]. Proceedings IEEE Symposium on Information Visualization. IEEE, 1996: 46-53.

Qu H, Wang H, Cui W, et al. Focus+ context route zooming and information overlay in 3D urban environments[J]. IEEE Transactions on Visualization and Computer Graphics, 2009, 15(6): 1547-1554.

Deng H, Zhang L, Mao X, et al. Interactive urban context-aware visualization via multiple disocclusion operators[J]. IEEE transactions on visualization and computer graphics, 2016, 22(7): 1862-1874.

Johansson M, Roupé M, Bosch-Sijtsema P. Real-time visualization of building information models (BIM)[J]. Automation in Construction, 2015, 54: 69-82.

Motamedi A, Hammad A, Asen Y. Knowledge-assisted BIM-based visual analytics for failure root cause detection in facilities management[J]. Automation in construction, 2014, 43: 73-83.

Islam S, Silver D, Chen M. Volume splitting and its applications[J]. IEEE Transactions on Visualization and Computer Graphics, 2007, 13(2): 193-203.

Juszczyk M, Tomana A, Bartoszek M. Current issues of BIM-based design change management, analysis and visualization[J]. Procedia engineering, 2016, 164: 518-525. 